\title{
TIMING IS UNPREDICTABLE AND THE SEVERITY IS UNCERTAIN: Removal Of Epidural Catheter After Postoperative Myocardial Infarction
}

\author{
Zeynep CAGIRAN, Nezih SERTOZ, Semra KARAMAN \\ Ege University Faculty of Medicine, Department of Anesthesiology \& Reanimation, Bornova, 35100 Izmir, \\ Turkey
}

\section{PURPOSE}

Myocardial infarction (MI) is one of the leading causes of perioperative morbidity and mortality. However management of the disease can be more complicated with both of the neuraxial techniques and anticoagulation therapy (1). We present a case of safe removal of epidural catheter 33 hours after oral dual anticaogulan and unfractionated heparin therapy with a normal coagulation function analysis before removal.

\section{CLINICAL FEATURES}

A 42 year old woman, weighing 85 kilograms, ASA 1, with no medical history underwent elective total hip arthroplasthy surgery under combined spinal epidural anaesthesia. She had no prior surgery. Her physical examination, laboratory and thyroid function tests and chest $X$ ray were normal. But due to inverted $\mathrm{T}$ waves in V1-4 on electrocardiogram, preoperative cardiology consultation was requested. Cardiology reported low risk for the patient's cardiac risk for surgery. The operation was uneventful. On arrival to postanesthesia care unit (PACU) her blood pressure was $95 / 65 \mathrm{mmHg}$ and pulse rate was 96 beats/min. She had a variegated skin without any chest pain. No inotropic support was required. Postoperative electrocardiogram revealed bigeminy ventricular extrasystole without ST segment elevation. Cardiac markers including troponin I were sent and cardiology doctor was called urgently. After the rising in troponin I levels, patient transferred to coronary intensive care unit with the diagnosis of non- st-segment elevation myocardial infarction. Dual oral antiplatelet therapy (asprin and thienopyridine ) and low molecular weight heparin (LMWH) were started.
Several hours later anesthesia consult was sought to evaluate for epidural catheter removal. The removal of epidural catheter in this patient required a clinical decision, balancing the risk of epidural hematoma with continuation of antiplatelet therapy against the risk of coronary re-thrombosis with discontinuation of the medication. Clopidogrel was then immediately discontinued in preparation for epidural catheter removal. The patient continued to receive subcutaneous LMWH twice a day with the last dose being 24 hours before the planned epidural catheter removal. On the second postoperative day after routine coagulation tests, the catheter was removed uneventfully. She was monitored in the surgical intensive care for two hours following catheter removal. She was discharged home postoperative 13th day in stable condition.

\section{CONCLUSION}

Timing for removal of epidural catheter in anticoagulated patients is still a very hard decision if further coagulation and aggregation tests are not available. Considering a reasonable balance of minimizing the risk of epidural hematoma and the risks of thromboembolic events, the catheter removal may very likely be less than 36 hours.

\section{References}

1. Glenn E, Mehl J, Rosinia FA, Liu H. Safe removal of an epidural catheter 72 hours after clopidogrel and aspirin administrations guided by platelet function analysis and thromboelastography. $\mathrm{J}$ Anaesthesiol Clin Pharmacol. 2013;1:99-101. 